\title{
A dynamic equilibrium of three hydrogen-bond conformers explains the NMR spectrum of the active site of photoactive yellow protein
}

\section{Supplementary Information}

Phillip Johannes Taenzler, Keyarash Sadeghian, and Christian Ochsenfeld*

\author{
Chair of Theoretical Chemistry, Department of Chemistry, \\ University of Munich (LMU), \\ Butenandtstr. 7, D-81377 München, Germany \\ and \\ Center for Integrated Protein Science (CIPSM) at the Department of Chemistry, \\ University of Munich (LMU), \\ Butenandtstr. 5-13, D-81377 München, Germany
}

(Dated: August 16, 2016)

\section{CONTENTS}

1Structural information regarding QM regions 2

2ZPE corrections

3NMR calculations on the crystal structure of PYP with deprotonated Arg52

$4 \mathrm{UV} / \mathrm{V}$ is vertical excitation energy calculations on the crystal structure of PYP

5Additional NMR Calculation for the QM/MM minimized structures

$6 \mathrm{NMR}$ Calculation on the QM/MM minimized T50V, E46Q, and Y42F mutants

7FF MD simulation on the conformers

8Investigation of possible Basis Set Superposition Error effects

9Structure files

References

* christian.ochsenfeld@uni-muenchen.de 


\section{Structural information regarding QM regions}

The QM regions were chosen to spread inter-residual interactions (competing hydrogen bonds, charged groups, $\pi$-stacking, etc) between Glu46-H, Tyr42-H, their donor and acceptors, and other residues over as many distinct QM regions as possible. The $\mathrm{QM}$ regions are defined as any residue having at least one atom within a given radius of $p$ CA's phenoxy atom. This radius was multiplied by $\sqrt[3]{2}$ from one QM region to the subsequent up to R6 in order to simulate a doubling of the volume of the contributing sphere around $p \mathrm{CA}$. To avoid going beyond the active region of the structural optimizations, the volume of R7 was chosen to only be $50 \%$ larger than that of R6. The identification of the residues is based on the crystal structure of PYP to avoid incorporating randomly placed water molecules in any QM region.

Table S1: Structural information, number of atoms, and residues included in the QM regions

\begin{tabular}{|c|c|c|c|}
\hline Selection & QM Atoms & Radius $[\AA]$ & Residues in QM Region \\
\hline $\mathrm{R} 1$ & 70 & 2.32 & $\mathrm{C} 69+p \mathrm{CA}, \mathrm{Y} 42, \mathrm{E} 46$ \\
\hline $\mathrm{R} 2$ & 107 & 2.92 & $\mathbf{R 1}+\mathrm{I} 31, \mathrm{~T} 50$ \\
\hline R3 & 129 & 3.69 & $\mathbf{R 2}+\mathrm{F} 62$ \\
\hline $\mathrm{R} 4$ & 155 & 4.65 & $\mathbf{R 3}+\mathrm{R} 52$ \\
\hline $\mathrm{R} 5$ & 248 & 5.85 & R4 + G47, V57, V66, A67, F96, V122 \\
\hline R6 & 425 & 7.37 & $\begin{array}{c}\text { R5 + G29, A30, I39, N43, A45, I49, } \\
\text { G51, T70, F75, M100, V105, V120 }\end{array}$ \\
\hline $\mathrm{R} 7$ & 517 & 8.43 & R6 + Q33, L33, N41, D48, D53, P54, V107 \\
\hline $\mathrm{QM}_{S}$ & 86 & - & $\mathrm{C} 69+p \mathrm{CA}, \mathrm{Y} 42, \mathrm{E} 46, \mathrm{~T} 50$ \\
\hline $\mathrm{QM}_{L}$ & 160 & - & $\mathrm{C} 69+p \mathrm{CA}, \mathrm{I} 31, \mathrm{Y} 42, \mathrm{E} 46, \mathrm{~T} 50, \mathrm{G} 51, \mathrm{R} 52, \mathrm{~F} 62$ \\
\hline $\mathrm{QM}_{X L}$ & 270 & - & $\begin{array}{c}\mathrm{C} 69+p \mathrm{CA}, \mathrm{G} 29, \mathrm{~A} 30, \mathrm{I} 31, \mathrm{Y} 42, \mathrm{~A} 45, \mathrm{E} 46, \mathrm{G} 47 \\
\mathrm{I} 49, \mathrm{~T} 50, \mathrm{G} 51, \mathrm{R} 52, \mathrm{~F} 62, \mathrm{~F} 96, \mathrm{~V} 120, \mathrm{~V} 122\end{array}$ \\
\hline
\end{tabular}




\section{ZPE corrections}

The zero-point energies (ZPE) of each conformer was determined for the smallest QM/MM setup (B3LYP-D/def2$\mathrm{SVP} / \mathrm{QM}_{S}$ ) based on numerically calculated harmonic frequencies of the QM region only. A comparison of the ZPE corrected and uncorrected relative energies of the conformers is shown in Table S2. As no significant changes in the relative energies were observed, no further ZPE corrections were carried out.

Table S2: ZPE corrected relative energies of the T, F, and G conformers

\begin{tabular}{c|c|c} 
& Uncorrected $\Delta \mathrm{E}\left[\mathrm{kcal} \mathrm{mol}^{-1}\right]$ & ZPE corrected $\Delta \mathrm{E}\left[\mathrm{kcal} \mathrm{mol}^{-1}\right]$ \\
\hline $\mathrm{T}$ & 0.00 & 0.00 \\
\hline $\mathrm{F}$ & 3.70 & 3.68 \\
\hline $\mathrm{G}$ & 2.20 & 1.87 \\
\hline
\end{tabular}




\section{NMR calculations on the crystal structure of PYP with deprotonated Arg52}

A schematic representation of Arg52, and nearby residues is shown in Figure S1. As Arg52 only enters the QM region starting at R4, the calculations were repeated from there with each of Arg52's five protons removed. The results are presented in Tables S3 and S4. The neutron diffraction structure shows HH12 to be absent [1].

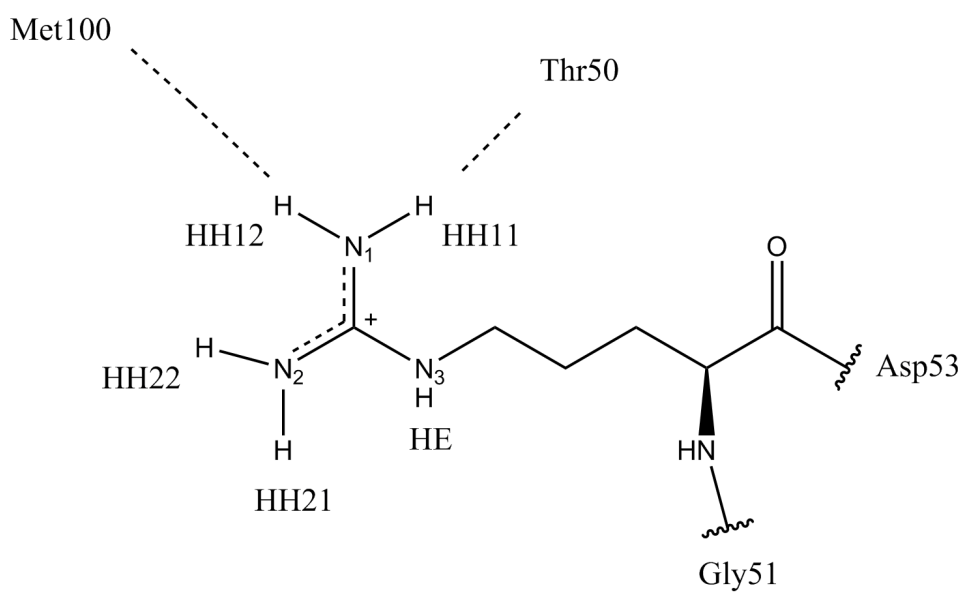

Figure S1: Schematic representation of R52, and all nearby residues

Table S3: The calculated B97-2/pcS-1 NMR shifts in ppm of Glu46-H based on the crystal structure with deprotonated Arg52. The calculated NMR shifts based on the protonated Arg52 system are included as a reference. All reported shifts are relative to TMS.

\begin{tabular}{|l|c|c|c|c|c|c|}
\hline QM Region & Protonated Arg52 & HH22 & HH21 & HH12 & HH11 & HE \\
\hline R4 & 19.97 & 20.14 & 20.14 & 20.13 & 20.14 & 20.14 \\
\hline R5 & 20.17 & 20.34 & 20.34 & 20.33 & 20.33 & 20.33 \\
\hline R6 & 20.24 & 20.42 & 20.42 & 20.41 & 20.42 & 20.42 \\
\hline R7 & 20.23 & 20.38 & 20.38 & 20.37 & 20.39 & 20.39 \\
\hline
\end{tabular}

Table S4: The calculated B97-2/pcS-1 NMR shifts in ppm of Tyr42-H based on the crystal structure with deprotonated Arg52. The calculated NMR shifts based on the protonated Arg52 system are included as a reference.

All reported shifts are relative to TMS.

\begin{tabular}{|c|c|c|c|c|c|c|}
\hline QM Region & Protonated Arg52 & HH22 & HH21 & HH12 & HH11 & HE \\
\hline R4 & 10.74 & 11.12 & 11.12 & 11.09 & 11.10 & 11.07 \\
\hline R5 & 10.80 & 11.15 & 11.15 & 11.13 & 11.14 & 11.10 \\
\hline R6 & 10.93 & 11.27 & 11.27 & 11.25 & 11.26 & 11.23 \\
\hline R7 & 10.97 & 11.31 & 11.31 & 11.29 & 11.31 & 11.28 \\
\hline
\end{tabular}




\section{UV/Vis vertical excitation energy calculations on the crystal structure of PYP}

Table S5: The lowest RICC2/AMBER vertical excitation energy computed for the crystal structure of PYP which corresponds to a local $\pi \rightarrow \pi^{*}$ transition within the $p$ CA chromophore (basis set aug-cc-pVDZ). The QM-regions R1 and R2 (in brackets) were chosen for these calculations.

\begin{tabular}{|l|c|c|c|}
\hline Structure & $\mathrm{S}_{0} \rightarrow \mathrm{S}_{1}[\mathrm{eV}]$ & Oscillator Strength & Reference Values $[\mathrm{eV}]$ \\
Crystal $[1]$ & $3.37(3.38)$ & 1.23 & $2.78[2,3]$ \\
\hline
\end{tabular}

The lowest QM/MM vertical excitation energy computed for the local excited state of PYP in the crystalline conformation shows very large deviation $(0.6 \mathrm{eV})$ from the experimentally measured absorption maximum structure. This can be explained by the fact that we only relaxed the position of those hydrogen atoms which were not assigned in the neutron diffraction structure. Furthermore, we did not include any solvent effects which may also influence the excitation energy. Overall, and as expected, the crystalline conformation does not provide a suitable basis to study spectroscopic data which are measured in solution at room temperature conditions. 
5. Additional NMR Calculation for the QM/MM minimized structures
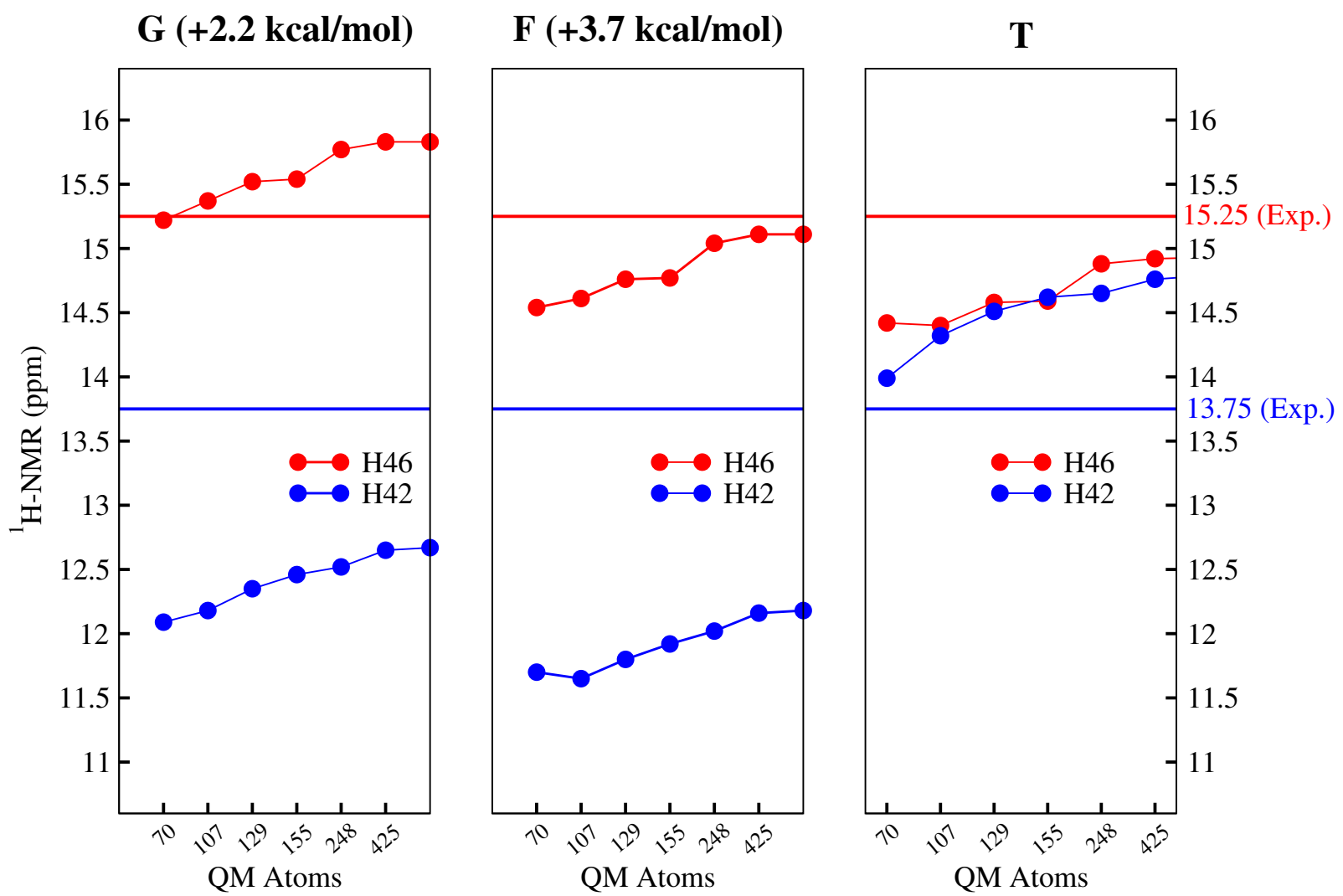

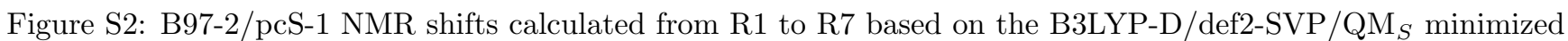
structures 

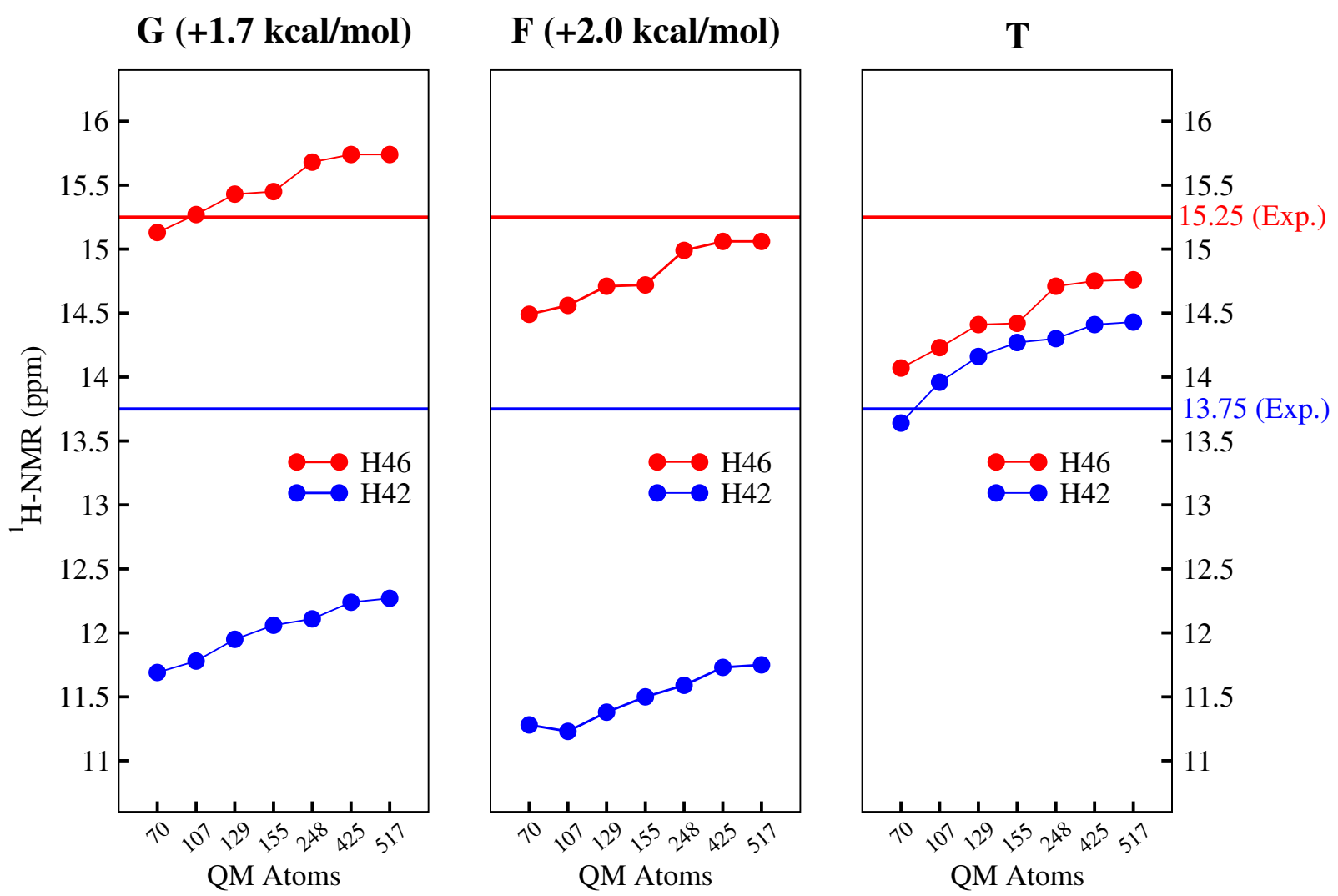

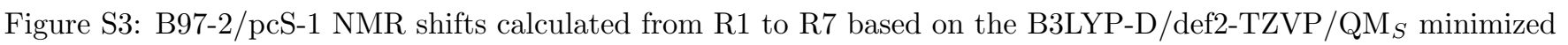
structures 

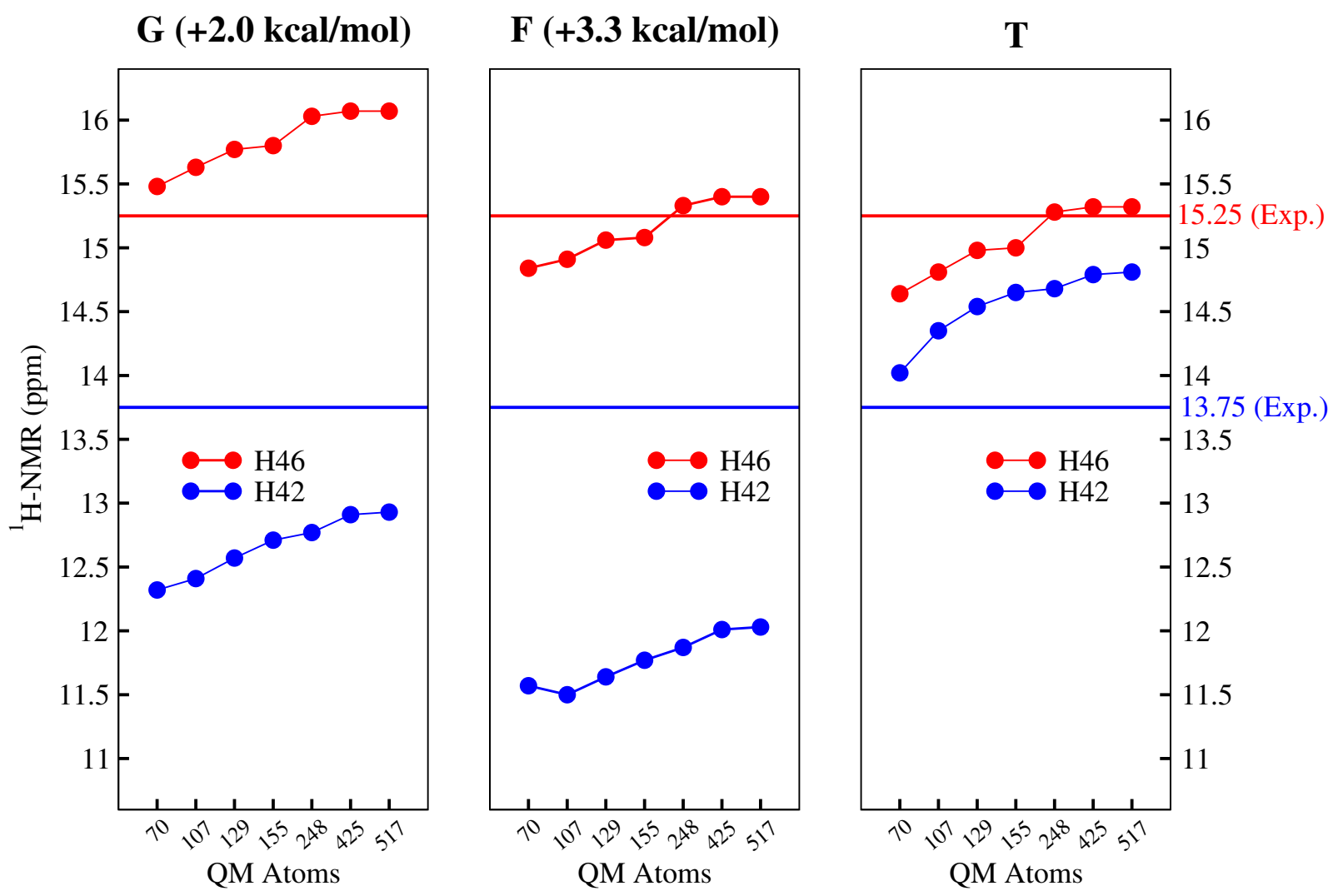

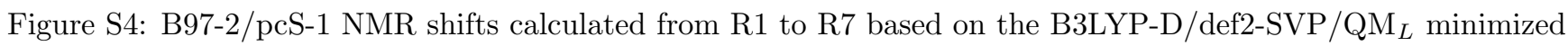
structures 
6. NMR Calculation on the QM/MM minimized T50V, E46Q, and Y42F mutants

\section{T50V Mutant}

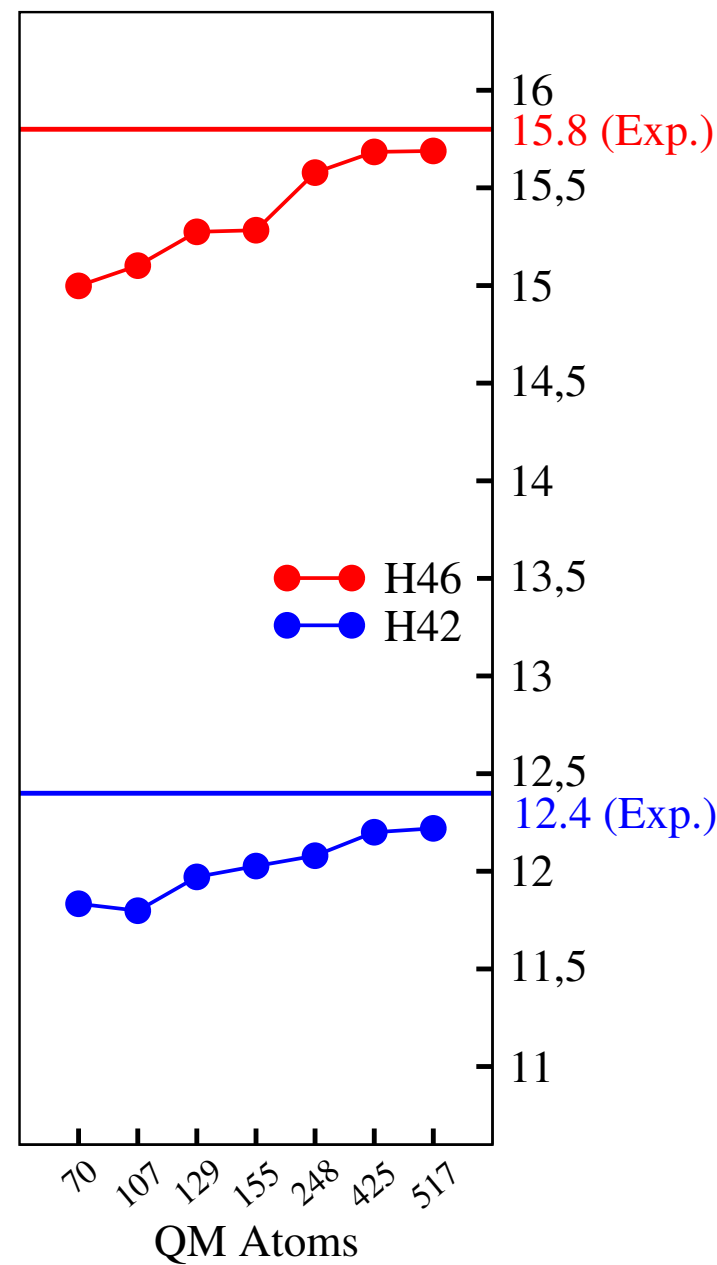

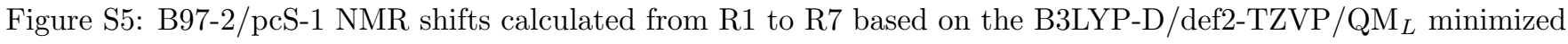
structure of $\mathrm{T} 50 \mathrm{~V}$ 

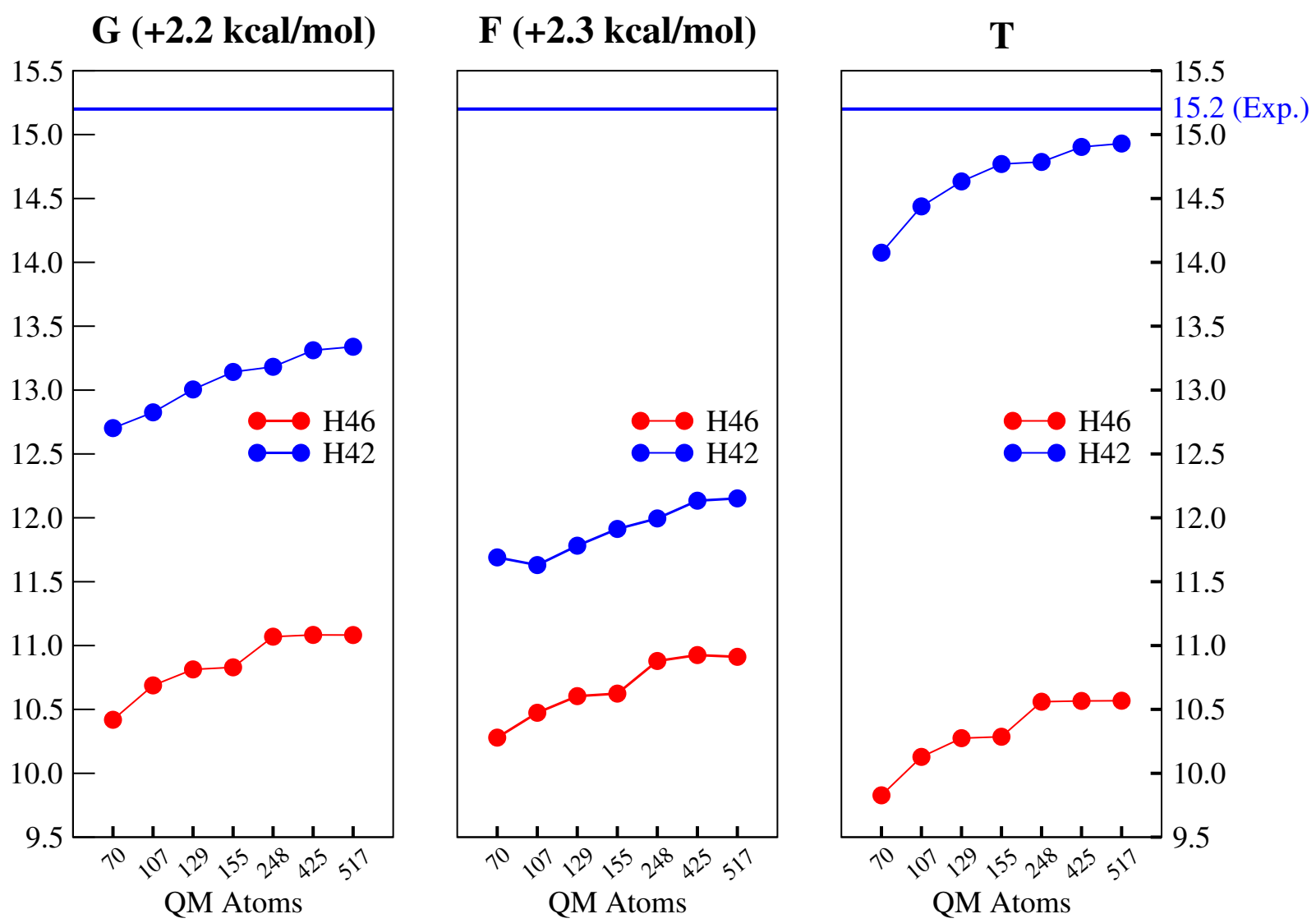

Figure S6: B97-2/pcS-1 NMR shifts calculated from R1 to R7 based on the B3LYP-D/def2-TZVP/QM ${ }_{L}$ minimized structures of E46Q 

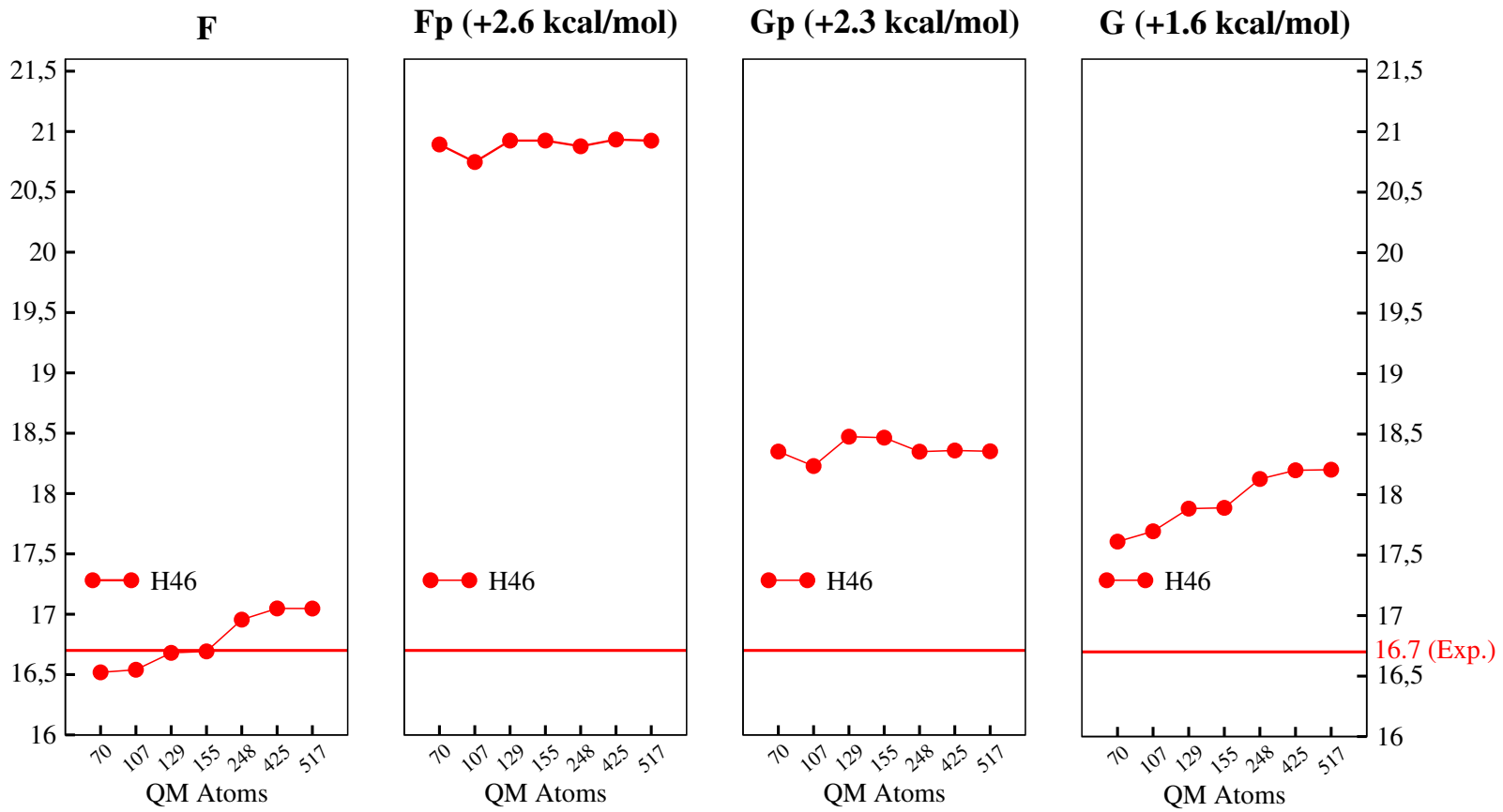

Figure S7: B97-2/pcS-1 NMR shifts calculated from R1 to R7 based on the B3LYP-D/def2-TZVP/QM ${ }_{X L}$ minimized structures of $\mathrm{Y} 42 \mathrm{~F}$ 


\section{FF MD simulation on the conformers}

In order to examine the effects of the mobile solvent, and the thermal motion of the protein on the NMR shifts of the hydrogen bond network, we performed force-field MD simulations, followed by QM/MM relaxations and NMR calculations. The residues of the smallest QM region ( $p$ CA+Cys69, Tyr42, Glu46, Thr50) were frozen in place for each conformer. Subsequent 5.5 ns MD simulations were performed for each conformer, with the first 500 ps being used for equilibration. The RMSD of the protein backbone of each simulation is shown in Figure S8. Starting at $500 \mathrm{ps}$, snapshots were taken in $200 \mathrm{ps}$ increments, for which QM/MM relaxations were performed at the B3LYPD/AMBER/def2-SVP level. The MD simulations were performed in the NVE ensemble, with a temperature of $298 \mathrm{~K}$, and a harmonic spherical potential of radius $35 \AA$ centered at V120. The time step was taken to be 1 fs. The QM region was taken to be $\mathrm{QM}_{L}$, with the active region being adjusted for each frame so as to include any residue having at least one atom within a $7.5 \AA$ radius of any of $p \mathrm{CA}+\mathrm{Cys} 69$ 's constituent atoms.
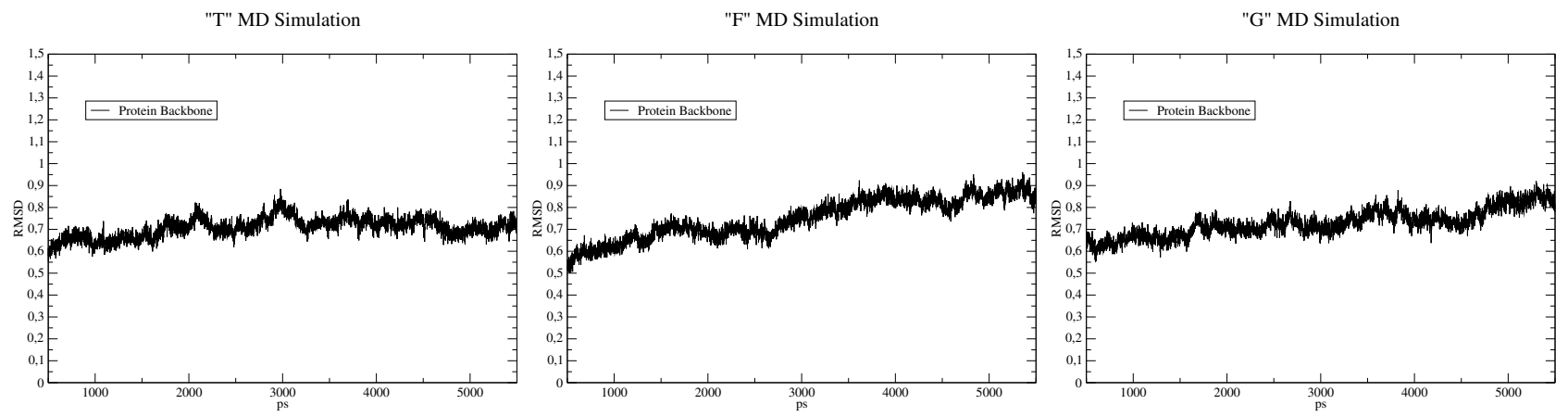

Figure S8: RMSD of the Protein Backbone of the "T" (left), "F" (center), and "G" (right) MD simulations

It was observed that 1 of the 26 "F", and 7 of the 26 "G" snapshots flipped into the "T" conformer during the QM/MM structural optimizations.

NMR calculations utilizing B97-2/AMBER/pcS-1 were subsequently performed on these structures utilizing the QM region $\mathrm{R} 6$. The results are shown in Table S6 The average NMR shift of each proton in each conformer run was calculated, along with the standard deviation. NMR shifts obtained from structures with flipped conformers (marked in red) were excluded from the averaging. 
Table S6: B97-2/pcS-1 NMR shifts calculated using R6 based on B3LYP-D/def2-SVP/QM ${ }_{L}$ structures obtained from FF MD simulations. NMR shifts corresponding to structures exhibiting conformation flip are shown in red, and are excluded from the averages shown below

\begin{tabular}{|c|c|c|c|c|c|c|}
\hline & \multicolumn{2}{|c|}{$\mathrm{T}$} & \multicolumn{2}{|c|}{$\mathrm{F}$} & \multicolumn{2}{|c|}{ G } \\
\hline Frame $[\mathrm{ps}]$ & Glu46-H & Tyr42-H & Glu46-H & Tyr42-H & Glu46-H & Tyr42-H \\
\hline 500 & 15.3 & 14.8 & 14.8 & 12.9 & 15.5 & 12.7 \\
\hline 700 & 14.6 & 15.0 & 15.0 & 15.2 & 15.5 & 13.1 \\
\hline 900 & 14.8 & 15.2 & 15.3 & 12.4 & 15.2 & 13.1 \\
\hline 1100 & 14.5 & 15.1 & 15.1 & 12.5 & 15.5 & 13.5 \\
\hline 1300 & 14.3 & 14.7 & 15.3 & 12.7 & 16.1 & 13.3 \\
\hline 1500 & 15.2 & 15.0 & 14.8 & 12.6 & 15.8 & 13.2 \\
\hline 1700 & 14.7 & 14.7 & 15.3 & 13.2 & 15.9 & 12.7 \\
\hline 1900 & 14.8 & 14.9 & 14.8 & 13.0 & 15.5 & 13.0 \\
\hline 2100 & 14.8 & 14.7 & 14.9 & 13.2 & 15.4 & 12.8 \\
\hline 2300 & 14.7 & 15.0 & 14.9 & 12.4 & 15.5 & 12.5 \\
\hline 2500 & 15.0 & 15.0 & 15.1 & 12.4 & 15.0 & 13.0 \\
\hline 2700 & 14.8 & 14.8 & 15.1 & 13.0 & 15.1 & 13.0 \\
\hline 2900 & 14.6 & 15.2 & 15.2 & 12.3 & 15.1 & 13.3 \\
\hline 3100 & 14.2 & 14.5 & 15.4 & 12.8 & 15.4 & 13.3 \\
\hline 3300 & 15.0 & 14.6 & 15.3 & 12.8 & 15.3 & 13.8 \\
\hline 3500 & 14.8 & 14.4 & 15.3 & 12.7 & 14.5 & 15.1 \\
\hline 3700 & 15.0 & 14.8 & 15.2 & 12.8 & 14.4 & 14.6 \\
\hline 3900 & 14.0 & 14.5 & 14.9 & 12.5 & 14.8 & 15.3 \\
\hline 4100 & 14.4 & 14.0 & 15.0 & 12.0 & 14.8 & 15.2 \\
\hline 4300 & 14.4 & 14.5 & 15.1 & 12.7 & 15.1 & 13.0 \\
\hline 4500 & 14.2 & 14.2 & 14.9 & 12.8 & 15.2 & 13.0 \\
\hline 4700 & 14.9 & 14.5 & 14.8 & 12.5 & 14.9 & 15.6 \\
\hline 4900 & 14.9 & 15.2 & 15.4 & 13.0 & 15.3 & 13.1 \\
\hline 5100 & 14.2 & 14.4 & 14.8 & 12.4 & 15.4 & 13.3 \\
\hline 5300 & 14.3 & 14.9 & 15.4 & 12.7 & 14.1 & 14.8 \\
\hline 5500 & 14.6 & 14.5 & 15.0 & 12.8 & 15.2 & 14.9 \\
\hline Avg & 14.6 & 14.7 & 15.1 & 12.7 & 15.4 & 13.1 \\
\hline Std. Dev. & 0.3 & 0.3 & 0.2 & 0.3 & 0.3 & 0.3 \\
\hline
\end{tabular}




\section{Investigation of possible Basis Set Superposition Error effects}

In order to investigate the presence and impact of BSSE effects on the relative energies of the three conformers, we performed additional RI-MP2/MM single-point calculations on the optimized structures using the same QM regions and basis sets as for the optimizations. The results in $\mathrm{kcal} \mathrm{mol}^{-1}$ are shown below:

\begin{tabular}{c|c|c|c|c} 
& $\mathrm{QM}_{S} /$ def2-SVP & $\mathrm{QM}_{S} /$ def2-TZVP & $\mathrm{QM}_{L} /$ def2-SVP & $\mathrm{QM}_{L} /$ def2-TZVP \\
\hline $\mathrm{T}$ & 0.0 & 0.0 & 0.0 & 0.0 \\
\hline $\mathrm{F}$ & +2.6 & +1.4 & +2.2 & +0.9 \\
\hline $\mathrm{G}$ & +2.6 & +1.9 & +2.1 & +1.9 \\
\hline
\end{tabular}

We next performed pure QM calculations, in which the residues of $\mathrm{QM}_{L}$ (excluding Gly51 due to its bond with Thr50 - this $\mathrm{QM}$ region is as denoted $\mathrm{QM}_{L}{ }^{\prime}$ ) not found in $\mathrm{QM}_{S}$ were replaced with ghost atoms in order to determine the impact of the BSSE. The resulting relative energies in $\mathrm{kcal} \mathrm{mol}^{-1}$ are shown below:

\begin{tabular}{c|c|c|c} 
& $\mathrm{QM}_{L}{ }^{\prime} / \operatorname{def} 2-\mathrm{TZVP}$ & $\mathrm{QM}_{S} /$ def2-TZVP & $\mathrm{QM}_{S}+$ ghost $/$ def2-TZVP \\
\hline $\mathrm{T}$ & 0.0 & +0.7 & +0.4 \\
\hline $\mathrm{F}$ & 0.0 & 0.0 & 0.0 \\
\hline $\mathrm{G}$ & +0.4 & +1.6 & +1.5 \\
\hline
\end{tabular}

Although there is a qualitative difference between the QM/MM and the pure QM calculations (the "F" conformer is more stable in the pure QM set), the actual differences in the relative energies are insignificant. Furthermore, there are essentially no differences in the relative energies with and without ghost atoms, indicating that the BSSE is negligible. 


\section{Structure files}

The largest QM/MM structural optimizations (B3LYP-D/def2-TZVP/QM ${ }_{L}$ ) for each conformer are attached as t-qmmm-opt.pdb, f-qmmm-opt.pdb, and g-qmmm-opt.pdb. 
[1] S. Yamaguchi, H. Kamikubo, K. Kurihara, R. Kuroki, N. Niimura, N. Shimizu, Y. Yamazaki, and M. Kataoka, Proceedings of the National Academy of Sciences 106, 440 (2009).

[2] A. D. Stahl, M. Hospes, K. Singhal, I. v. Stokkum, R. v. Gronelle, M. L. Groot, and K. J. Hellingwerf, Biophysical Journal 101, 1184 (2011).

[3] Y. Imamoto, M. Kataoka, and F. Tokunaga, Biochemistry 35, 14047 (1996). 